\title{
On the one extremal problem on the Riemann sphere
}

\author{
A. Targonskii ${ }^{1, a *}$, I. Targonskaya ${ }^{2, b}$ \\ ${ }^{1}$ Department of Mathematical Analysis, Zhytomyr State University, Velyka Berdychivs'ka, 40, 10008 \\ Zhytomyr, Ukraine \\ a targonsk@mail.ru, targonsk@zu.edu.ua
}

Keywords: Inner radius of domain, quadratic differential, piecewise-separating transformation, Green function, radial systems of points, logarithmic capacity, variational formula.

\begin{abstract}
Sharp estimates of product of inner radii for pairwise disjoint domains are obtained. In particular, we solve an extremal problem in the case of arbitrary finite number of rays containing arbitrary even number of free poles.
\end{abstract}

\section{Introduction}

This paper belongs to the theory of extremal problems on classes of non-overlapping domain, which is a separate direction in geometric theory of functions of a complex variable. The begin of these investigations associated with the paper of M. A. Lavrent'ev [1] in 1934. He found the maximum of some functional with respect to two simply connected domains with two fixed points. We note that this result was needed him for applying to some aerodynamics problems. In 1947, G. M. Goluzin solved a similar problem for three fixed points on the complex plane [2]. Then the topic began to evolve rapidly. In this connection we may recall papers of many authors, including Y. E. Alenitsina, M. A. Lebedev, J. Jenkins, P. M. Tamrazov, P. P. Kufareva and others. Using the idea of P. M. Tamrazov, in 1975 G. P. Bakhtin solved first the problem with so-called "free poles" on the unit circle, see, e.g., [3].

An important step for the development of this topic was papers of V. N. Dubinin. He developed a new method of research that is method of piecewise-separating transformation. He also first solved numerous of extremal problems for an arbitrary but fixed multi connected non-overlapping domains (see, e.g., [4], [5], [6]). Now this type of extremal problems is used for investigations in holomorphic dynamics.

In the last decade actively used Bakhtin's method of "managing functional". He managed to solve a series of extremal problems for so-called "radial systems of points" (see, e.g., [4], [7], [8], [9], [10]). In the present paper we use the mentioned about Bakhtin's method.

\section{Theory}

Let $\mathbb{N}, \mathbb{R}$ and $\mathbb{C}$ be the sets of natural, real and complex numbers respectively. Let $\overline{\mathbb{C}}:=\mathbb{C} \bigcup\{\infty\}$ be the Riemannian sphere and $\mathbb{R}^{+}:=(0, \infty)$.

We fix a number $n \in \mathbb{N}$. A system of points $A_{n}=\left\{a_{k} \in \mathbb{C}: k=\overline{1, n}\right\}$, we will called the $n$-equiangular system of points on rays, if for all $k=\overline{1, n}$, the following relations are satisfied:

$$
\arg a_{k}=\frac{2 \pi}{n}(k-1) \text {. }
$$

We introduce the following "managing" functionals for an arbitrary $n$-equiangular system of points on rays 


$$
\mathcal{L}^{(\alpha)}\left(A_{n}\right)=\left[\prod_{k=1}^{n} \chi\left(\left|\frac{a_{k}}{a_{k+1}}\right|^{\frac{n}{4}}\right)\right]^{1-\frac{2 \alpha}{n^{2}}} \cdot\left[\prod_{k=1}^{n}\left|a_{k}\right|\right]^{1+\frac{\alpha}{n}}
$$

where $\chi(t)=\frac{1}{2}\left(t+\frac{1}{t}\right), t \in \mathbb{R}^{+}$.

Let us consider a system of angular domains:

$$
P_{k}:=\left\{w \in \mathbb{C}: \frac{2 \pi}{n}(k-1)<\arg w<\frac{2 \pi}{n} k\right\}, \quad k=\overline{1, n} .
$$

Let $\left\{B_{k}, B_{\infty}\right\}$ be an arbitrary non-overlapping domains such that

$$
a_{k} \in B_{k}, \infty \in B_{\infty}, \quad B_{k}, B_{\infty} \subset \overline{\mathbb{C}}, \quad k=\overline{1, n}
$$

Let

$$
g_{B}(B, a)=h_{B, a}(z)+\log \frac{1}{|z-a|}
$$

generalized Green's function of domains $B$ with respect to a point $a \in B$. If $a=\infty$, then

$$
g_{B}(B, \infty)=h_{B, \infty}(z)+\log \frac{1}{|z|} \text {. }
$$

The value of

$$
r(B, a):=\exp \left(h_{B, a}(z)\right)
$$

the define of inner radius domain $B \subset \overline{\mathbb{C}}$ with respect to a point $a \in B$ (see [4], [5], [6], [11], [12], [13]).

We use the concept of a quadratic differential. Recall that a quadratic differential on a Riemann surface $S$ is a map

$$
\varphi: T S \rightarrow \mathbb{C}
$$

satisfying

$$
\varphi(\lambda v)=\lambda^{2} \varphi(v)
$$

for all $v \in T S$ and all $\lambda \in \mathbb{C}$. If $z \in U \rightarrow \mathbb{C}$, is a chart defined on some open set $U \subset S$ then $\varphi$ is equal on $U$ to

$$
\varphi_{U}(z) d z^{2}
$$

for some function $\varphi_{U}$ defined on $z(U)$.

Suppose that two charts $z: U \rightarrow \mathbb{C}$ and $w: V \rightarrow \mathbb{C}$ on $S$ overlap, and let

$$
h:=w \circ z^{-1}
$$

be the transition function. If $\varphi$ is represented both as $\varphi_{U}(z) d z^{2}$ and $\varphi_{V}(w) d w^{2}$ on $U \cap V$, then we have

$$
\varphi_{V}(h(z))\left(h^{\prime}(z)\right)^{2}=\varphi_{U}(z) .
$$

One way to say this is that quadratic differentials transform under pull-backs by the square of the derivative. As the main results associated with it can be found in [14]. 


\section{Results}

In this paper we investigate the following problems.

Problem. Let $n \in \mathbb{N}, n \geq 2, \alpha \leq n^{2}, \alpha \in \mathbb{R}^{+}$. We intend to find a maximum of the functional

$$
r^{\alpha}\left(B_{\infty}, \infty\right) \cdot \prod_{k=1}^{n} r\left(B_{k}, a_{k}\right)
$$

and to describe all its extremals, if $A_{n}$ be an arbitrary $n$-equiangular system of points on rays satisfying the condition (1), and $\left\{B_{k}, B_{\infty}\right\}$ be an arbitrary set of non-overlapping domains, $a_{k} \in B_{k}, \infty \in B_{\infty}$, $B_{k}, B_{\infty} \subset \overline{\mathbb{C}}, k=\overline{1, n}$.

Theorem. Let $n \geq 2, n \in \mathbb{N}, \alpha \in \mathbb{R}^{+}, \alpha \leq n^{2}$. Let also $A_{n}=\left\{a_{k}\right\}_{k=1}^{n}$ be an n-equiangular system of points on rays, and $B_{\infty}, B_{k} \subset \overline{\mathbb{C}}, k=\overline{1, n}, a_{k} \in B_{k}, \infty \in B_{\infty}$ be an arbitrary set of non-overlapping domains. Then we have the inequality

$$
r^{\alpha}\left(B_{\infty}, \infty\right) \prod_{k=1}^{n} r\left(B_{k}, a_{k}\right) \leq r^{\alpha}\left(B_{\infty}^{(0)}, \infty\right) \prod_{k=1}^{n} r\left(B_{k}^{(0)}, a_{k}^{(0)}\right) .
$$

The equality sign holds, if points $\left\{a_{k}\right\}$ and domains $B_{k}, B_{\infty}$ are the poles and the circular domains of the quadratic differential

$$
Q(w) d w^{2}=-w^{n-2} \frac{n^{2}+\left(w^{n}-1\right) \alpha}{\left(w^{n}-1\right)^{2}} d w^{2},
$$

where $\mathcal{L}^{(\alpha)}\left(A_{n}\right)=1$.

When $\alpha=n^{2}$ we obtain the result follows.

Corollary. Let $n \geq 2, n \in \mathbb{N}, \alpha \in \mathbb{R}^{+}, \alpha \leq n^{2}$. Let also $A_{n}=\left\{a_{k}\right\}_{k=1}^{n}$ be an n-equiangular system of points on rays, and $B_{\infty}, B_{k} \subset \overline{\mathbb{C}}, k=\overline{1, n}, a_{k} \in B_{k}, \infty \in B_{\infty}$ be an arbitrary set of non-overlapping domains. Then we have the inequality

$$
r^{n^{2}}\left(B_{\infty}, \infty\right) \prod_{k=1}^{n} r\left(B_{k}, a_{k}\right) \leq r^{n^{2}}\left(B_{\infty}^{(0)}, \infty\right) \prod_{k=1}^{n} r\left(B_{k}^{(0)}, a_{k}^{(0)}\right) .
$$

The equality sign holds, if points $\left\{a_{k}\right\}$ and domains $B_{k}, B_{\infty}$ are the poles and the circular domains of the quadratic differential

$$
Q(w) d w^{2}=-\frac{w^{2 n-2}}{\left(w^{n}-1\right)^{2}} d w^{2}
$$

where $\mathcal{L}^{(\alpha)}\left(A_{n}\right)=1$.

Proof of Theorem. The proof leans on a method of the piece-dividing transformation developed by V. Dubinin (see [4], [5], [6]).

The function

$$
z_{k}(w)=-i w^{\frac{n}{2}}
$$

realizes univalent and conformal transformations of domain $P_{k}$ on the right half-plane $\operatorname{Re} z>0$ for all $k=\overline{1, n}$.

Let $\omega_{k}^{(1)}:=z_{k}\left(a_{k}\right), \omega_{k-1}^{(2)}:=z_{k-1}\left(a_{k}\right), a_{n+1}:=a_{1}, \omega_{0}^{(2)}:=\omega_{n}^{(2)}, z_{0}:=z_{n}(k=\overline{1, n})$.

The family of functions $\left\{z_{k}(w)\right\}_{k=1}^{n}$ is a piece-dividing transformation (see [4], [5], [6]) of the domains $\left\{B_{k}: k=\overline{1, n}\right\}$ with respect to the system of angles $\left\{P_{k}\right\}_{k=1}^{n}$. For any domain $\Delta \in \mathbb{C}$ we define $(\Delta)^{*}:=\left\{w \in \overline{\mathbb{C}}: \frac{1}{\bar{w}} \in \Delta\right\}$. We denote by $\Omega_{k}^{(1)}$ the connected component 


$$
z_{k}\left(B_{k} \bigcap \bar{P}_{k}\right) \bigcup\left(z_{k}\left(B_{k} \bigcap \bar{P}_{k}\right)\right)^{*}
$$

containing a point $\omega_{k}^{(1)}$, and by $\Omega_{k-1}^{(2)}$ we denote the connected component

$$
z_{k-1}\left(B_{k} \bigcap \bar{P}_{k-1}\right) \bigcup\left(z_{k-1}\left(B_{k} \bigcap \bar{P}_{k-1}\right)\right)^{*}
$$

containing a point $\omega_{k-1}^{(2)}, k=\overline{1, n}, \bar{P}_{0}:=\bar{P}_{n}, \Omega_{0}^{(2)}:=\Omega_{n}^{(2)}$. Generally speaking, the domains $\Omega_{k}^{(s)}$ are multiconnected domains, $k=\overline{1, n}, s=1,2$. The pair of domains $\Omega_{k-1}^{(2)}$ and $\Omega_{k}^{(1)}$ is result of piece-dividing transformation of domains $B_{k}$ concerning families $\left\{P_{k-1}, P_{k}\right\},\left\{z_{k-1}, z_{k}\right\}$ at a point $a_{k}, k=\overline{1, n}$. We denote by, too, $\Omega_{k}^{(\infty)}$ the connected component

$$
z_{k}\left(B_{\infty} \bigcap \bar{P}_{k}\right) \bigcup\left(z_{k}\left(B_{\infty} \bigcap \bar{P}_{k}\right)\right)^{*}
$$

containing a point $\infty$. The system of domains $\Omega_{k}^{(\infty)}$ is result of piece-dividing transformation of domains $B_{\infty}$ concerning families $\left\{P_{k}\right\},\left\{z_{k}\right\}$ at a point $\infty, k=\overline{1, n}$.

From the formula (3) we obtain the following asymptotic expressions:

$$
\left|z_{k}(w)-z_{k}\left(a_{m}\right)\right| \sim \frac{n}{2} \cdot\left|a_{m}\right|^{\frac{n}{2}-1} \cdot\left|w-a_{m}\right|, \quad w \rightarrow a_{m}, w \in \bar{P}_{k}, \quad m=k, k+1 .
$$

From the Theorem 1.9 [11] (see also [5], [6]) and the formulae (4), we have the inequalities

$$
\begin{gathered}
r\left(B_{k}, a_{k}\right) \leqslant \frac{2}{n} \cdot\left(\frac{r\left(\Omega_{k}^{(1)}, \omega_{k}^{(1)}\right) \cdot r\left(\Omega_{k-1}^{(2)}, \omega_{k-1}^{(2)}\right)}{\left(\left|a_{k}\right|\left|a_{k+1}\right|\right)^{\frac{n-2}{2}}}\right)^{\frac{1}{2}}, \quad k=\overline{1, n}, \\
r\left(B_{\infty}, \infty\right) \leqslant \prod_{k=1}^{n}\left(r\left(\Omega_{k}^{(\infty)}, \infty\right)\right)^{\frac{2}{n^{2}}} .
\end{gathered}
$$

Using the inequality (5), we get the following relations: 


$$
\begin{aligned}
& r^{\alpha}\left(B_{\infty}, \infty\right) \prod_{k=1}^{n} r\left(B_{k}, a_{k}\right) \leq \\
& \leq\left(\frac{2}{n}\right)^{n} \cdot \prod_{k=1}^{n}\left(r^{\frac{4 \alpha}{n^{2}}}\left(\Omega_{k}^{(\infty)}, \infty\right) \cdot \frac{r\left(\Omega_{k}^{(1)}, \omega_{k}^{(1)}\right) \cdot r\left(\Omega_{k-1}^{(2)}, \omega_{k-1}^{(2)}\right)}{\left(\left|a_{k}\right|\left|a_{k+1}\right|\right)^{\frac{n-2}{2}}}\right)^{\frac{1}{2}}= \\
& =\left(\frac{2}{n}\right)^{n} \cdot \prod_{k=1}^{n} \frac{\left(\left|a_{k}\right|^{\frac{n}{2}}+\left|a_{k+1}\right|^{\frac{n}{2}}\right)^{1-\frac{2 \alpha}{n^{2}}} \cdot\left(\left|a_{k}\right|^{\frac{n}{2}} \cdot\left|a_{k+1}\right|^{\frac{n}{2}}\right)^{\frac{2 \alpha}{n^{2}}}}{\left(\left|a_{k}\right|\left|a_{k+1}\right|\right)^{\frac{n}{4}}}\left|a_{k}\right|= \\
& \times \prod_{k=1}^{n}\left(r^{\frac{4 \alpha}{n^{2}}}\left(\Omega_{k}^{(\infty)}, \infty\right) \cdot \frac{r\left(\Omega_{k}^{(1)}, \omega_{k}^{(1)}\right) \cdot r\left(\Omega_{k-1}^{(2)}, \omega_{k-1}^{(2)}\right)}{\left|\omega_{k}^{(1)}-\omega_{k}^{(2)}\right|^{2-\frac{4 \alpha}{n^{2}}} \cdot\left(\left|\omega_{k}^{(1)}\right|\left|\omega_{k}^{(2)}\right|\right)^{\frac{4 \alpha}{n^{2}}}}\right)^{\frac{1}{2}}= \\
& =\left(\frac{2}{n}\right)^{n} \cdot \prod_{k=1}^{n}\left|a_{k}\right|^{1+\frac{\alpha}{n}} \cdot \prod_{k=1}^{n}\left(\frac{\left|a_{k}\right|^{\frac{n}{2}}+\left|a_{k+1}\right|^{\frac{n}{2}}}{\left(\left|a_{k}\right|\left|a_{k+1}\right|\right)^{\frac{n}{4}}}\right)^{1-\frac{2 \alpha}{n^{2}}} \times \\
& \times \prod_{k=1}^{n}\left(\frac{r^{\frac{4 \alpha}{n^{2}}}\left(\Omega_{k}^{(\infty)}, \infty\right) \cdot r\left(\Omega_{k}^{(1)}, \omega_{k}^{(1)}\right) \cdot r\left(\Omega_{k-1}^{(2)}, \omega_{k-1}^{(2)}\right)}{\left|\omega_{k}^{(1)}-\omega_{k}^{(2)}\right|^{2-\frac{4 \alpha}{n^{2}}} \cdot\left(\left|\omega_{k}^{(1)}\right|\left|\omega_{k}^{(2)}\right|\right)^{\frac{4 \alpha}{n^{2}}}}\right)^{\frac{1}{2}}= \\
& =\left(\frac{2}{n}\right)^{n} \cdot 2^{n-\frac{2 \alpha}{n}} \cdot \mathcal{L}^{(\alpha)}\left(A_{n}\right) \times \\
& \times \prod_{k=1}^{n}\left(\frac{r^{\frac{4 \alpha}{n^{2}}}\left(\Omega_{k}^{(\infty)}, \infty\right) \cdot r\left(\Omega_{k}^{(1)}, \omega_{k}^{(1)}\right) \cdot r\left(\Omega_{k-1}^{(2)}, \omega_{k-1}^{(2)}\right)}{\left|\omega_{k}^{(1)}-\omega_{k}^{(2)}\right|^{2-\frac{4 \alpha}{n^{2}}} \cdot\left(\left|\omega_{k}^{(1)}\right|\left|\omega_{k}^{(2)}\right|\right)^{\frac{4 \alpha}{n^{2}}}}\right)^{\frac{1}{2}} .
\end{aligned}
$$

Functional

$$
\frac{r^{\alpha_{1}}\left(B_{1}, a_{1}\right) \cdot r^{\alpha_{2}}\left(B_{2}, a_{2}\right) \cdot r^{\alpha_{3}}\left(B_{3}, a_{3}\right)}{\left|a_{1}-a_{2}\right|^{\alpha_{1}+\alpha_{2}-\alpha_{3}} \cdot\left|a_{1}-a_{3}\right|^{\alpha_{1}-\alpha_{2}+\alpha_{3}} \cdot\left|a_{2}-a_{3}\right|^{-\alpha_{1}+\alpha_{2}+\alpha_{3}}},
$$

$a_{k} \in B_{k} \subset \overline{\mathbb{C}}, B_{k} \cap B_{\underline{p}}, k \neq p, \alpha_{k} \in \mathbb{R}^{+}, k, p=1,2,3$, relatively invariant conformal automorphisms of the complex plane $\overline{\mathbb{C}}([4],[15])$.

Taking into account the ratio of the last assertion of from (6), we have:

$$
\begin{gathered}
r^{\alpha}\left(B_{\infty}, \infty\right) \prod_{k=1}^{n} r\left(B_{k}, a_{k}\right) \leq\left(\frac{2}{n}\right)^{n} \cdot \mathcal{L}^{(\alpha)}\left(A_{n}\right) \\
\times \prod_{k=1}^{n}\left(r^{\frac{4 \alpha}{n^{2}}}\left(G_{k}^{(\infty)}, \infty\right) \cdot r\left(G_{k}^{(1)},-i\right) \cdot r\left(G_{k-1}^{(2)}, i\right)\right)^{\frac{1}{2}} \\
=\left(\frac{2}{n}\right)^{n} \cdot \mathcal{L}^{(\alpha)}\left(A_{n}\right) \cdot\left(r^{\frac{4 \alpha}{n^{2}}}\left(D_{\infty}, \infty\right) \cdot r\left(D_{1},-i\right) \cdot r\left(D_{2}, i\right)\right)^{\frac{n}{2}},
\end{gathered}
$$

where $D_{\infty}, D_{1}, D_{2}$ - the circular domains of the quadratic differential

$$
Q(z) d z^{2}=\frac{n^{2}-\alpha\left(1+z^{2}\right)}{\left(1+z^{2}\right)^{2}} d z^{2} .
$$


In the quadratic differentials (8) make the change from the formula (3).

$$
\begin{aligned}
Q(w) d w^{2} & =\frac{n^{2}-\alpha\left(1+\left(-i w^{\frac{n}{2}}\right)^{2}\right)}{\left(1+\left(-i w^{\frac{n}{2}}\right)^{2}\right)^{2}}\left(-i \frac{n}{2} w^{\frac{n}{2}-1}\right)^{2} d w^{2}= \\
& =\frac{n^{2}+\alpha\left(w^{n}-1\right)}{\left(w^{n}-1\right)^{2}}\left(-\frac{n^{2}}{4}\right) w^{n-2} d w^{2} .
\end{aligned}
$$

We obtain the differential (2).

Using the relation (7) we finally obtain

$$
r^{\alpha}\left(B_{\infty}, \infty\right) \prod_{k=1}^{n} r\left(B_{k}, a_{k}\right) \leq r^{\alpha}\left(B_{\infty}^{(0)}, \infty\right) \prod_{k=1}^{n} r\left(B_{k}^{(0)}, a_{k}^{(0)}\right),
$$

where points $\left\{a_{k}\right\}$ and domains $B_{k}, B_{\infty}$ are the poles and the circular domains of the quadratic differential (2). The theorem is proved.

Acknowledgement. The author is grateful to Prof. Bakhtin for suggesting problems and useful discussions.

\section{References}

[1] Lavrent'ev M.A. On the theory of conformal mappings. Tr. Fiz.-Mat. Inst. Akad. Nauk SSSR. 1934, (5), 159-245. (in Russian)

[2] Goluzin G.M. Geometric theory of functions of a complex variable. Nauka, Moscow, 1966. (in Russian)

[3] Bakhtina G.P. Variational methods and quadratic differentials in problems for disjoint domains. PhD thesis, Kiev, 1975. (in Russian)

[4] Bakhtin A.K., Bakhtina G.P., Zelinskii Yu.B. Topological-algebraic structures and geometric methods in complex analysis. Inst. Math. NAS Ukraine, Kiev, 2008. (in Russian)

[5] Dubinin V.N. Separating transformation of domains and problems of extremal division. Zap. Nauchn. Sem. Leningrad. Otdel. Mat. Inst. Ros. Akad. Nauk. 1988, (168), 48-66. (in Russian)

[6] Dubinin V.N. Method of symmetrization in the geometric theory of functions of a complex variable. Usp. Mat. Nauk. 1994, 49 1(295), 3-76.

[7] Bakhtin A.K. Inequalities for the inner radii of nonoverlapping domains and open sets. Ukr. Math. J. 2009, 61 (5), 716-733.

[8] Bakhtin A.K., Targonskii A.L. Extremal problems and quadratic differentials. Nonlin. Oscillations. 2005, 8 (3), 296-301.

[9] Targonskii A.L. Extremal problems of partially nonoverlapping domains on a Riemann sphere. Dop. NAN Ukr. 2008, (9), 31-36. (in Russian)

[10] Targonskii A.L. Extremal problems on the generalized $(n ; d)$-equiangular system of points. Anal. St. 2014, XXII (2), 239-251.

[11] Dubinin V.N. Asymptotic representation of the modulus of a degenerating condenser and some its applications. Zap. Nauchn. Sem. Peterburg. Otdel. Mat. Inst. 1997, (237), 56-73. (in Russian) 
[12] Dubinin V.N. Capacities of condensers and symmetrization in geometric function theory of complex variables. Dal'nayka, Vladivostok, 2009. (in Russian)

[13] Hayman W.K. Multivalent functions. Cambridge University, Cambridge, 1958.

[14] Jenkins J.A. Univalent functions and conformal mapping. Springer, Berlin, 1958.

[15] Kolbina L.I. Conformal mapping of the unit circle into nonoverlapping domains. Vest. Lening. Univ. 1995, 5, 37-43. (in Russian) 